Boise State University

ScholarWorks

5-1-2009

\title{
Conceptual Development About Motion and Force in Elementary and Middle School Students
}

Dewey I. Dykstra

Boise State University

Dale R. Sweet

Walter Strom Middle School 


\title{
Conceptual development about motion and force in elementary and middle school students $^{\dagger}$
}

\author{
Dewey I. Dykstra, Jr. \\ ddykstra@boisestate.edu \\ Department of Physics, Boise State University, Boise, Idaho 83725-1570 \\ and \\ Dale R. Sweet \\ sweetd@cleelum.wednet.edu \\ Walter Strom Middle School, 2694 State Route 903, Cle Elum, Washington 98922
}

\begin{abstract}
Methods of physics education research were applied to find what kinds of changes in 4th, 6th, and 8th grade student understanding of motion can occur and at what age. Such findings are necessary for the physics community to effectively discharge its role in advising and assisting pre-college physics education. Prior to and after instruction the students were asked to carefully describe several demonstrated accelerated motions. Most pre-instruction descriptions were of the direction of motion only. After instruction, many more of the students gave descriptions of the motion as continuously changing. Student responses to the diagnostic and to the activity materials revealed the presence of a third "snapshot" view of motion not discussed in the literature. The 4th and 6th grade students gave similar pre-instructional descriptions of the motion, but the 4th grade students did not exhibit the same degree of change in descriptions after instruction. Our findings suggest that students as early as 6th grade can develop changes in ideas about motion needed to construct Newtonian-like ideas about force. Students' conceptions about motion change little under traditional physics instruction from these grade levels through college level.
\end{abstract}

\footnotetext{
$\dagger$ Funded by NSF grant MDR-9145015. Opinions expressed herein are not necessarily those of the National Science Foundation.
} 


\section{Introduction}

By late elementary school students have formed implicit conceptions of their physical world. These conceptions formed early in their lives remain largely unchanged as a result of traditional instruction. ${ }^{1}$ We know that significant numbers of high school and college students can construct more powerful conceptions of physical phenomena, but most do not in response to traditional physics instruction. ${ }^{1}$ One of the issues raised by this observation is how early can students be engaged in such conceptual change. The findings in this article suggest some responses to this issue.

The physics community serves as a resource to the pre-college education community. We are consulted about textbook and curriculum issues, teach physics to those preparing to teach, and teach courses and workshops for pre-college teachers. These activities are sufficiently important that the American Association of Physics Teachers has established standing committees on Physics in the Pre-High School and High School levels and Physics Teacher Preparation. How can the physics community properly carry out its responsibilities in this area if we do not know how to help young students develop new understandings of physical phenomena, what understandings can be developed, and at what age ${ }^{2,3}$ This article presents one response to this important question.

\section{Children's conceptions of the physical world}

Students' conceptions about the physical world have been studied by the physics education research community for about three decades. ${ }^{4}$ We have had some success in teaching high school and college students to revise their conceptions of physical phenomena. ${ }^{5-9}$ Although there has been some work with pre-high school students' understanding of motion, we wonder 

http://www.kzoo.edu/ajp/4. Copyright restrictions may apply.

what revisions younger students can make in their initial conceptions as a result of the type of instruction found to be effective with older students. ${ }^{10-12}$

Before the development of the physics education research community, studies of pre-high school children's conceptions concerning motion were conducted as early as the second quarter of the 20th century. Conducting interviews with children aged $5-16$, Piaget and his colleagues explored children's notions of physical causality as they explained the motion of objects. ${ }^{10}$ They found the children's explanations developed from the use of animism and moral necessity by the younger children to explanations we observe in high school and college today. ${ }^{1,6-8}$ Cross and Pithkey ${ }^{11}$ have shown that young children associate higher speeds with closer objects, which can have disastrous consequences in decisions about crossing streets. Twigger and colleagues ${ }^{12}$ studying children aged $10-15$ corroborate findings by Piaget in the older children. Twigger and colleagues also found little evidence for change in these conceptions. We take the lack of change as evidence that these conceptions about motion are formed not so much in response to schooling, but in response to experience with moving objects and immersion in a culture (including schooling) whose members function with similar conceptions of motion and that schooling apparently does little to change their conceptions.

\section{Affecting children's conceptions about motion}

The use of microcomputer-based laboratories (MBL) promises important instructional advantages in the study of motion by students as indicated in the work of Brasell. ${ }^{5}$ Brasell studied differences in junior high aged students that arise as a result of studying motion via "realtime" generated graphs of one's own motion (non-delayed MBL) in comparison to the same activities in which the display of the graphs of motion were delayed by just 20 - $30 \mathrm{~s}$ after the 
motion was completed (delayed MBL) and a control group who did paper-and-pencil activities

(no MBL). Pre and post diagnostic responses revealed that the use of non-delayed-MBL accounted for $90 \%$ of the significant improvement over the delayed MBL whose performance did not show a significant improvement over the no MBL group.

\section{The Objective of Our Work}

Our goal is to go beyond documenting students' conceptions and to focus on questions necessary to make informed curriculum design decisions. For example:

- What are the aspects of understanding motion that are necessary to begin to develop a Newtonian view of forces?

- Under what conditions may we expect this understanding to be developed by children?

- To what extent may we expect these ideas to be developed by children in various grades?

In our study we did not look at graph interpretation skills, in which the students did engage, or at mathematical skills, which were unnecessary. We looked instead for evidence of change in conceptions about motion on which such skills might be built with understanding, instead of by rote drill.

Experience with an approach to forces and motion developed originally by Minstrell ${ }^{17,18}$ and described by Dykstra ${ }^{7}$ strongly suggests that students, for whom the changing motion process does not have high status in their thoughts about moving objects, do not distinguish between acceleration and velocity as physicists understand these two concepts. ${ }^{8,14,19,20}$ In the everyday view, acceleration is just another word for velocity or speed, in the particular case of 
This is an author-produced, peer-reviewed version of this publication. The final, definitive version of this document can be found online at the American Journal of Physics (doi: 10.1119/1.3090824) published by American Institute of Physics, at: http://www.kzoo.edu/ajp/4. Copyright restrictions may apply.

increasing speed. As a result they are less likely to realize or need to distinguish between an everyday view of forces and a Newtonian-like view of force. The Newtonian-like view of force rests specifically on acceleration as physicists know it, whereas the everyday view of force rests on velocity.

We used instruction based on the Tools for Scientific Thinking (TST) Project. ${ }^{6}$ We studied the extent to which students in the 4th, 6th, and 8th grades distinguish between speed and changing speed, in contrast to change or difference in speed, in their observations of motion, and the extent to which this distinction changes as a result of instruction.

Previous work such as that of Brasell ${ }^{5}$ and the classroom experience of one of the authors (Sweet) suggest that the 4th through 8th grade is an appropriate place in which to look. Because we are looking for evidence of what change in thinking about the kinematics is possible, their work is informative, but does not directly answer the curriculum development questions we consider necessary. It is evident that people do not usually advance the status of changing motion in their thinking, either spontaneously or due to standard instruction. ${ }^{1,21,22}$ Hence, if physicists wish that students develop a Newtonian-like view of force, it is important to devise a pedagogy and curriculum that is effective at inducing such conceptual change and to determine when it can be deployed.

\section{Experimental Design}

We administered a pre-instruction diagnostic on motion, then conducted the instruction on motion, followed by a re-administration of the diagnostic. This procedure was followed once with 4th grade students and twice each in a different academic year with 6th and 8th grade students. We looked for evidence of similarities and differences on the pre- and post-instruction diagnostics and in changes from pre- to post-instruction diagnostics. 

http://www.kzoo.edu/ajp/4. Copyright restrictions may apply.

Sweet conducted the instruction with the assistance of Dykstra. Sweet was the classroom teacher of the 6th grade students. We worked with the 4th and 8th grade students through cooperative arrangements with their teachers. We worked with all the students in the school at each grade level. All data is for students present for both pre- and post-diagnostics, matched pairs (see Table 1).

The TST instructional material, modified as described in the following, engages the students in examining graphs of their own motion. ${ }^{6}$ The graphs are produced on a computer display as the students move (non-delayed-MBL). Each type of graph, position-time, velocitytime, and acceleration-time, was introduced in laboratory activities. All students worked in groups of four or five in the 6th grade classroom; each group had a computer equipped with an ultrasonic motion detector. In these sessions they carried out different activities such as the following:

1. Each person is asked to make a particular motion. From this the group has to decide what features on the resulting graphs relate to particular features of the motion.

2. If you follow these directions for making a motion, what would a graph of that motion look like? Test your conclusions.

3. How would you have to move to make the graph shown? Make up your own mind, then decide as a group and test your conclusions.

Students were challenged in homework and class discussion to go from verbal descriptions of motions to graphs of these motions and vice versa. The emphasis throughout was not who "got it right" first, but on questions such as: What do you think? Why do you think so? Did it turn out the way you thought? What implications do the results have for what you 
originally thought? What do features in each type of graph depict about motion? Can we use our conclusions to match the graphs that the computer makes?

We devised a group-administered, demonstration interview with free-form responses to detect how students "see" motion for three examples of accelerated motion. The class is shown an example. Then each student is asked to describe the motion as completely and carefully as possible before moving to the next example. Each motion is repeated as often as the students ask. The individual student descriptions are generated in free form on paper without discussion. The three examples were the following:

1. A bean bag is allowed to fall to a horizontal surface, but is not allowed to bounce, which we label as “drop."

2. A can of refried beans is given a gentle push up an incline and is allowed to roll up and back down, which we label "incline."

3. A ball on the end of a string (about $60 \mathrm{~cm}$ long) is allowed to swing from left to right and back through one complete (but only one) oscillation, which we label "swing."

We asked students to concentrate on the time from just after the release to just before the motion was stopped in each case.

The criteria for a complete, careful description were left up to the students. Such criteria were not discussed with the students before, during, or after the study. Students did ask about the criteria. Our response was "We are really interested in what you think. Write what makes sense to you in a way that you think someone else reading it would understand." At no time were these descriptions from the diagnostic evaluated, discussed, or given back to the students. 
In the activities students occasionally wrote motion descriptions for their own use when directed in the materials. The instruction did not involve evaluation or discussion of the form of these writings. The students participated in activities involving motion very similar to the second one used in the diagnostic. The fact that this "up and down the ramp" motion was studied serves as an indicator of the effect of studying a particular motion on student descriptions of the motion in contrast to their descriptions of motion not specifically studied during the instruction.

The TST materials were intended for high school and college age students. The instructions given in the worksheets, how to run the software, and manipulate the apparatus were rewritten for younger students. ${ }^{23}$ The students did all of the activities in the TST motion materials, which were offered to the students in small or single function blocks so that the activities could be more easily done. The TST worksheets (readability level: grades 10-13) were revised for a readability level of mid-seventh grade level as computed by the Flesch-Kincaid method. $^{39}$

\section{Learning Results}

\section{A. Overall Summary}

The descriptions of motion that students gave in the diagnostic, both before and after instruction, can be categorized into three groups.

1. One type of answer is characterized as: "It went that-a-way." The direction of the motion was all that was mentioned; for example, "It fell down," "It rolled up and then down," "It swung left and then right." In this type of answer what is important is whether there is motion or not and in what direction.

2. We describe the second type of answer as a "snapshot." ${ }^{24}$ In this type of answer the direction is usually mentioned, as is the speed or velocity, but never with reference to the 
This is an author-produced, peer-reviewed version of this publication. The final, definitive version of this document can be found online at the American Journal of Physics (doi: 10.1119/1.3090824) published by American Institute of Physics, at: http://www.kzoo.edu/ajp/4. Copyright restrictions may apply.

change of motion process itself. These answers looked like: "It fell down fast," "It rolled up fast and then down slow," and "It swung left slow then fast and then to the right slow then fast." There was no mention of speeding up or slowing down as continuous processes. Any speed changes are referred to only in terms of differences or relative size. It is as if the change process is not important, just the speed before and after.

3. The third type of answer usually mentions the direction of motion, but also includes reference to whether or not there was continuous change in magnitude. Answers of this type looked like: "It sped up as it fell," or "It fell down and didn't change its speed," "It rolled up slowing down and back down speeding up," "It swung left speeding up and then slowing down and then it swung right speeding up and then slowing down." What is important is not whether the students were able to detect acceleration, in free fall for example, but whether they looked for and decided whether there was continuous change.

This last way of "seeing" motion is important before students are likely to benefit from the type of lessons on force that are effective at affecting conceptual change. ${ }^{7,8,20}$

Initially we expected to see evidence of two views: direction-only and dynamic. Our first attempts at categorizing the students' writing on the pre-diagnostic in terms of only these two views were problematic. We independently categorized the student papers, but there were examples for which we did not agree. The problematic student papers were ones that mentioned different speeds without directly addressing the change itself in any way. The solution was to introduce an intermediate category, the snapshot category. What led us to add the snapshot category and its pedagogical significance are described in the following.

The groups represented in Figs. 1-3 were considered typical by their teachers and are a more cautious basis for drawing conclusions. The teachers at the school agreed that the original 
This is an author-produced, peer-reviewed version of this publication. The final, definitive version of this document can be found online at the American Journal of Physics (doi: 10.1119/1.3090824) published by American Institute of Physics, at: http://www.kzoo.edu/ajp/4. Copyright restrictions may apply.

6th and 8th grade groups who were our subjects in the first trials were better than average academically. These same two groups made bigger changes in our testing, so we labeled them as "higher performance" in Figs. 4 and 5. The data taken as a whole illustrate the range that might be expected with students in these grades.

Figures 1-3 in the pre-test items suggest that the preponderance of the responses of the 4th and 6th grade students were in the direction-only and snapshot type categories, whereas the responses of the 8th grade students were more evenly distributed across the categories on the pre-diagnostic tasks. This difference in distribution between the 4th and 6th grade students and 8th grade students might be explained by the fact that all of the 8th grade students in this study (see Figs. 3 and 5) had already experienced a traditional unit of instruction on motion and force. However, the 8th grade students exhibited dynamic type responses on only about $50 \%$ of the pretest tasks. This low performance after traditional instruction is also seen in the TST project with older students. ${ }^{6}$

In our study not quite $50 \%$ of the 4 th grade students (see Fig. 1) gave a dynamic description on any task in the post-diagnostic. The task on which more demonstrated this description was the second task on the diagnostic, similar to one in the instructional activities. Although the 6th grade students (Fig. 2) gave a pre-test performance little different than the 4th grade students, their performance on post-diagnostic tasks had noticeably more dynamic-type responses than the 4th grade group. If we compare Figs. 2 and 3 and Figs. 4 and 5, the 6th grade students to the 8th grade students, we see that about the same percentages of the 6th grade students did the same as the 8th grade students in the end. That is, the advantage of experiencing traditional instruction by the 8th grade students was minimal after using the modified TST materials. 

http://www.kzoo.edu/ajp/4. Copyright restrictions may apply.

The percentage of students giving a direction-only type answer decreased for all students on all tasks from pre to post diagnostic. Only in the 4th grade group (see Fig. 1) did this sort of answer increase on the drop task and remain the same on the incline task. We see that there is a clear trend away from direction-only type responses toward dynamic-type responses. The TST based experience appears to result in students changing their understanding of motion in all of the classes, albeit to differing degrees.

\section{B. The snapshot view: challenge to our original expectations}

When we started this work we thought only in terms of whether or not students had a sufficiently differentiated view of motion. Did the students look for the process of change in the motion? During the instruction with the first group of students (6th grade, Fig. 4), we noticed that students had trouble with the intent of instructions to move with changing speed such as:

"Start from rest, walk away from the detector while speeding up ..." and "Repeat your motion several times until the velocity graph shows a smooth, steady increase in velocity..."

The students would walk with a steady velocity or would walk slowly and then suddenly walk with a greater speed. When coaching each other how to move, they would say things like, "Walk slow and then go fast."

Because we had encountered most college students having problems at the same point in their activities, we realized that the problem may not be due to the instructions being poorly written. ${ }^{25}$ Instead, we realized we were encountering the very issue that we had intended to engage in the instruction. On the pre-diagnostic tasks most students seem not to attend to the process of change in motion. Hence, it should be no surprise that continuous change in motion is not obvious to the students in our lab instructions or in their attempts to produce the movements. 
This is an author-produced, peer-reviewed version of this publication. The final, definitive version of this document can be found online at the American Journal of Physics (doi: 10.1119/1.3090824) published by American Institute of Physics, at: http://www.kzoo.edu/ajp/4. Copyright restrictions may apply.

At best they might notice the velocity is different at different times, a kind of snapshot view of motion that changes.

With this new interpretation, we looked back at the pre-diagnostic responses. We found that we independently agreed on the categorization of each response, if we added a snapshot-type classification.

The evidence for the snapshot view suggests that the observation of speed differences is important. It could be argued that this observation is a step toward the dynamic view and away from the direction-only view. If so, we would expect a person expressing the snapshot view on the pre-diagnostic would be more likely to have changed to the dynamic view or remain at the snapshot view on the post-diagnostic than move to the direction-only view on the postdiagnostic. We find that this expectation is the case as illustrated in Table 2.

If we take the descriptions they give as indicative of how they are "seeing" motion, it is the case that students initially holding the snapshot view were shifted to the dynamic view in greater numbers than not changing or moving to the direction-only view by the end of instruction. They are also more likely to remain in the snapshot view than move to the directiononly view. This unidirectional tendency in the changes in the numbers in each category suggests a sequential nature to the views with the snapshot view representing an intermediate position on motion between the direction-only and the dynamic views.

To what extent is our interpretation concerning the snapshot view supported by our observations of college students? We checked on a prediction made by the college students at the same point in their studies of motion using the TST materials. The college students were nonscience majors in a conceptual physics course. After studying distance (position) graphs and velocity graphs, they were asked to predict "If you made a motion in front of the detector 
This is an author-produced, peer-reviewed version of this publication. The final, definitive version of this document can be found online at the American Journal of Physics (doi: 10.1119/1.3090824) published by American Institute of Physics, at: http://www.kzoo.edu/ajp/4. Copyright restrictions may apply.

consisting of standing at rest for one second, walking away from the detector while speeding up for about one and one half seconds and then walking away while slowing down for about one and one half seconds, to a stop and standing at rest for one second, what would the velocity graphs look like?"

Figure 6 illustrates the majority responses of 99 students to this question. Eight students made three additional predicted velocity graphs not shown. Figure 6(a) generated by only 15 students is one we would like all students to sketch by the end of the unit of study. It illustrates a constantly increasing speed during the first part of the motion and then a constantly decreasing speed during the second part of the motion. Another graph was similar, but lacked distinct straight lines sloping up and down and a distinct transition from speeding up to slowing down. It could be argued that this second prediction, seen in Fig. 6(b) and drawn by 21 students, is the result of less specific notions about the nature of the changes in the motion than those held by students who drew Fig. 6(a). The third prediction supports the existence of the snapshot view. Figure 6(c), drawn by 40 students, consists of an interval of constant velocity, punctuated by sudden changes in magnitude. There also appear to be associations of speeding up with larger velocity and of slowing down with smaller velocity. The notion of continuous change in velocity seems not to have occurred to these students even in the context of the phrases used in the instructions. There is clear evidence that "stop" may imply an almost instantaneous change to zero velocity rather than a process of slowing down to a stop.

The fact that 40 of the 99 students made the prediction illustrated in Fig. 6(c) supports our conjecture that the snapshot construct should be taken into account in kinematics instruction and that such a view is resistant to traditional instruction. Traditional instruction at best probably enables students to move to the intermediate, snapshot view and not beyond, but we do not have 

http://www.kzoo.edu/ajp/4. Copyright restrictions may apply.

evidence to support this conjecture. All of the students in the course had physical science instruction including motion and force in junior high and perhaps at some time earlier in elementary school. A few students in the college course had high school physics.

At this point in their study these students had spent time in lab and class discussion studying constant velocity motion. Some of the students suggested that such work in their previous laboratory activities might be why they did not draw sloping lines on the graphs. It can also be argued that the focus on constant velocity in the early part of the unit reinforced an outlook on motion the students were already using; an outlook in which constantly increasing or decreasing speed was of low status. ${ }^{19}$ Because of the low status of these ideas, the students did not notice them even in specific instructions. The students apparently constructed their own meanings for the instructions, rather than that intended by the authors of the materials.

We wonder why continuously changing speed is of low status based on everyday experience. For most people most of the time, our focus of attention is to reach some speed and then maintain it until we achieve our destination. In everyday experience we usually need to pay little attention to the details of getting up to speed or stopping. The start up and stopping portion of the motion while walking and driving is usually of short duration compared to the total time traveled. Thus, why should we expect the idea of continuously changing speed as a process itself to be cued by phrases such as "speeding up" or "slowing down" either for the purpose of predicting a graph or for making such a motion?

\section{Applying a New View of Motion to Force}

We worked with one group of the 6th grade students on force. This group's motion diagnostic performance is shown in Fig. 2. Our approach was patterned after the lessons briefly 
described in the appendix of the article by Dykstra, Boyle and Monarch ${ }^{7}$ and more extensively in narrative form by Dykstra. ${ }^{8}$ These lessons are the result of Dykstra's previous work mentored by Jim Minstrell. ${ }^{7,8}$

There were similarities and differences in the ways that 6th grade students approached the ideas compared to ways that high school and college students approach the same ideas. The 6th grade students decided that a constant force is not a good explanation for a constant velocity because they found experimentally that a constant force results in a constantly increasing velocity. They then focused on the same alternatives as did the older students for an explanation.

Both groups decided to consider a decreasing force as the explanation for a constant velocity. At this point both groups have collected data for different constant forces on the same nearly frictionless object. So, they all have access to data that showed the larger the constant force, the larger the acceleration of the object. Many of the older students reason their way through this idea based on their data to conclude that for any force, there is an acceleration. A decreasing force, the older students reason, will most probably give a decreasing acceleration, not a constant velocity. They decide the conclusion must be that zero force is the only reasonable alternative remaining. Finally, the older students struggle with what zero force means. ${ }^{8}$

Although the 6th grade students first seized on the decreasing force explanation for constant velocity, they did not note the significance of their data. Had we been able to spend more time with them or another group of students, we might have found a class who reasoned their way through this issue in a similar manner to the older students. Our 6th grade students needed to actually try a decreasing force to see what would happen.

We set up an apparatus in which a cart was pulled through a pulley system (a modified Atwood machine) along a horizontal surface by a falling cup full of water. The cup had a hole in 
the bottom through which water was allowed to flow once the apparatus was released to move. The 6th grade students devised this method to test their conjecture about decreasing force. They reasoned that if water were running out of the cup during the run, the pull by the string on the cart would decrease during the run. They expected to find a constant velocity as a result. Instead, the students decided that they could see in the force and acceleration graphs a decreasing force resulting in a decreasing acceleration, not a constant velocity. ${ }^{26}$ They decided a constantly decreasing force is not a good explanation for constant velocity. A zero force was the only the condition remaining they could imagine that produces a constant velocity, a realization not comfortably accepted.

This group of 6th graders was not the higher performance group whose motion diagnostic results are found in Fig. 4. This fact gives us greater confidence that it is reasonable to engage 6th grade students in thinking about force once they have developed a dynamic view of motion.

\section{Physics Education Research: challenges in the elementary classroom}

\section{A. Time allocation}

When one decides to teach from our perspective, the time necessary to allow students to negotiate "taken-as-shared" meaning often exceed the 30-minute (or less) time normally allotted

for a particular lesson. ${ }^{34}$ Long time periods are normally considered beyond the attention span of the students. Yet, our students were usually not ready to quit when the time was up in these longer periods. This instruction engages the students. It effectively uses longer blocks of time and involves considering things in greater depth.

\section{B. Contrasting classroom cultures}



http://www.kzoo.edu/ajp/4. Copyright restrictions may apply.

Both younger students and college students in are well habituated in the prevailing classroom culture. This culture evolves as students progress through school. Each age has its own challenges when asked to function differently. For the younger students the security of one's place in the hierarchy of one's cohorts and the authority of teachers are strong factors. For older students there is not usually an established cohort until they are well into the courses for the major, but there are well-established habits that have enabled success in surviving the traditional classroom experience. These habits usually do not involve speaking freely about one's ideas.

In the traditional classroom knowledge comes from the teacher. Typical student behavior is aimed at guessing what the teacher wants said and eliciting cues, clues, or validation from the teacher. Cautious students speak only when they are sure they are right. Many wait for the "smart kids" to speak first, because as soon as what the teacher wanted is said, the teacher moves on.

Because our interest is in student understanding, the classroom culture we attempted to create is different. We want our students' verbal behavior to focus on their own and their peers' ideas and on how these ideas either fit or do not fit experimental outcomes. We want the students to consider what they have seen and heard, sifting through it all, and talking with each other about what makes the most sense. The students can and do make good decisions about this. The teacher is not the source of explanatory knowledge or of its validation. Some readers may believe that elementary students cannot do this sort of abstract thinking. However, the 4th, 6th, and 8th grade students in our study discussed the issues in effective ways. ${ }^{35}$

A major problem is the mismatch of classroom cultures. Neither the students nor their teachers in this study were openly antagonistic to the culture we attempted to establish, but they 
were not practiced at it. Had they been experienced at the different classroom culture we were attempting, more might have been accomplished in the time available.

\section{Challenges and opportunities for teachers}

In the traditional classroom culture students either answer a specific question from the teacher or ask the teacher a question who answers the question directly and immediately. In the alternative classroom culture the focus is on getting students to talk about their ideas.

Teachers need to be careful in such settings. Students are so accustomed to look to the teacher for validation that they may perceive the teacher's answering a question with another question as a teacher's unwillingness to help the student. This perception may be passed on to other students, teachers, administrators, and parents, thus damaging a teacher's reputation as a willing and helpful teacher.

In the present study we needed to think about the nature of students' existing conceptions and what experiences might challenge aspects of these existing conceptions. The marking of papers in the project was not merely right or wrong, and included analysis of the evidence for students' ideas and our own performance. This analysis was based on our initial understanding of the students' ideas, how that compared with our classroom observations, evidence of change in understanding, and our perceptions concerning whether even more change could occur.

\section{Alternative pedagogies}

Is the alternative pedagogy and curriculum used in the study superior to what is normally available to students? The 8th grade students shifted their thinking about motion as a result of the experience in the study even though they had already experienced the traditional instruction on 
motion and force. The 6th grade performance after the study was similar to that of the 8th grade students even though the 6th grade students started closer to the 4th graders. Of the college students who had not yet experienced this pedagogy, $40 \%$ were still functioning consistently with the snapshot view. This evidence strongly supports an answer in the affirmative. It appears reasonable to accomplish with 6th and 8th grade students the kind of conceptual change we can achieve with high school and college students using this pedagogy.

\section{Discussion}

\section{A. Significance of the snapshot view of motion}

We observed an intermediate view of motion, the snapshot view, in which the velocities or speeds of an object at different times are compared without specific attention to the continuous change process itself in the magnitude of the speed. ${ }^{28}$ This snapshot view explains the difficulty that students of a different ages have with instructions to move with a continuously changing speed. ${ }^{30} \mathrm{We}$ must attend to this issue if our instruction is to be beneficial. This snapshot view is indicative of the well-known difficulty that students have learning the concept of acceleration in traditional instruction. ${ }^{31}$ It is not realistic to expect most of our students "hear" our meaning when we use phrases like "speeding up," "increasing velocity," or "decreasing velocity."

Adding the snapshot view as a category improved our ability to interpret student responses to the diagnostics and during instruction. In the snapshot view changes are noted in the form of differences only. Table 2 supports the notion that students initially using that view are more likely to be found using the dynamic view after instruction than using the direction-only view. 
This is an author-produced, peer-reviewed version of this publication. The final, definitive version of this document can be found online at the American Journal of Physics (doi: 10.1119/1.3090824) published by American Institute of Physics, at: http://www.kzoo.edu/ajp/4. Copyright restrictions may apply.

We see evidence of the snapshot view in students from 4th grade to college seniors. Traditional instruction on motion experienced by students between 4th grade and the end of college does little to change the snapshot view once it is developed. The snapshot view indicates a low status for continuously changing motion or velocity in a person's thinking. It is a barrier to changing from everyday thinking about force to a Newtonian-like view of force.

Acknowledging that a significant number of students hold this view also has pedagogical implications. We should not merely pressure students to "follow directions" or continue to search for a better wording of the instructions. ${ }^{32}$ We can engage students in closely examining their world to eventually find the limitations of this view themselves. The Motion unit of the Powerful Ideas in Physical Science materials ${ }^{33}$ uses this strategy and has been shown to be very effective at inducing conceptual change about motion. ${ }^{20,27}$

\section{B. Force in the curriculum}

Some insight as to how these students deal with issues relating to forces was gained. We saw that Newtonian views of forces might be attained by the 6th and 8th grade students, but that they might need to reason more from direct experiment than is necessary for some high school seniors or college students.

Given that some 4th grade students came to a dynamic view of motion, but not in large percentages, we would hesitate to recommend that the kind of issues on forces we discussed be taken up by all 4 th grade students. ${ }^{7,8}$ Continued work with such students on an experimental basis is justified. 


\section{Conclusions}

We studied changes in descriptions by 4th, 6th, and 8th grade students of three different accelerated motions. The 103 students in this study were typical of their age group. We believe that the results will be consistent in replications of this work.

Whether reaching a dynamic view of motion is a reasonable curricular goal for a majority of 4th grade students is unclear. Our results show that some 4th grade students do develop this dynamic view in response to instruction. Could the fact that the materials were at the mid-7th grade reading level have had any effect on the 4th grade students' performance? Might the 4th grade performance on the diagnostic more closely match that of the 6th and 8th grade students if the materials were modified or the 4th grade students were more used to the approach? These are questions we hope will be answered in the a future.

The data suggest we may expect that most 6th and 8th grade students are able to arrive at a dynamic view of motion. We attempted to engage the 6th grade students, in examining the nature of force once they had developed a dynamic view of motion. These students began to develop a Newtonian-like view of force on a developmental pathway similar to that observed in older students. ${ }^{7,8}$ Hence, a Newtonian-like view of force may be a reasonable curricular goal for students as early as the 6th grade.

\section{Acknowledgements}

We thank the students from whom we learned so much. We are also grateful for the cooperation and support of the teachers and administrators who helped make the study possible. Thanks to comments from two anonymous referees, the manuscript was greatly improved. We appreciate 
the opportunity to conduct this study with funding from the National Science Foundation (MDR-

9145015).

${ }^{1}$ R. Duit, Bibliography: Students' and Teachers' Conceptions and Science Education (Institute for Science Education (IPN) University of Kiel, Kiel, Germany 2007), <www.ipn.unikiel.de/aktuell/stcse/stcse.html>.

${ }^{2}$ D. I. Dykstra, Jr., “Science education in elementary school: Some observations,” J. Res. Sci. Teaching 24 (2), 179-182 (1987).

${ }^{3}$ D. I. Dykstra, Jr, “Author's response to comments of Cronin, Charron, and Espinet,” J. Res. Sci. Teaching 24 (7), 679-682 (1987).

${ }^{4}$ Although there are entries in Ref. 1 as far back as 1903, the vast majority of the entries are from after about $1978 .^{1}$

${ }^{5} \mathrm{H}$. Brasell, "The effect of real-time laboratory graphing on learning graphic representations of distance and velocity," J. Res. Sci. Teaching 24 (4), 385-395 (1987).

${ }^{6}$ R. K. Thornton and D. R. Sokolof, "Learning motion concepts using real-time microcomputerbased laboratory tools,” Am. J. Phys. 58 (9), 858-867 (1990).

${ }^{7}$ D. I. Dykstra, Jr., C. F. Boyle, and I. A. Monarch, "Studying conceptual change in learning physics," Sci. Educ. 76 (6), 615-652 (1992).

${ }^{8}$ D. I. Dykstra, Jr., “Teaching introductory physics to college students,” in Constructivism, Foundations, Perspectives and Practice, edited by Catherine Fosnot (Teachers College Press, New York, 2005), 2nd ed., pp. 182-204.

${ }^{9}$ L. C. McDermott, “Oersted Medal Lecture 2001: 'Physics education research - The key to student learning,'” Am. J. Phys. 69 (11), 1127-1137 (2001).

${ }^{10}$ J. Piaget, The Child's Conception of Physical Causality (Transaction Publishers, Brunswick, NJ, 2001) (originally published in French in 1927).

${ }^{11}$ R. T. Cross and A. Pithkey, "Speed, education and children as pedestrians: a cognitive change approach to a potentially dangerous naive concept.” Int. J. Sci. Educ. 10 (5), 531-540 (1988).

${ }^{12}$ D. Twigger, M. Byard, R. Driver, S. Draper, R. Hartley, S. Hennessy, R. Mohamed, C. O’Malley, T. O’Shea, and E. Scanlon, “The conception of force and motion of students aged 

http://www.kzoo.edu/ajp/4. Copyright restrictions may apply.

between 10 and 15 years: An interview study designed to guide instruction.” Int. J. Sci. Educ. 16 (2), 215-229 (1994).

${ }^{13}$ R. K. Thornton, "Tools for scientific thinking: Microcomputer-based laboratories for the naïve science learner," unpublished paper presented at the 7th National Educational Computing Conference, San Diego, CA (1986).

${ }^{14}$ Minstrell's work contain explanations of this approach and its application to other topics in physics. $^{15-18}$

${ }^{15} \mathrm{~J}$. Minstrell, "The role of the teacher in making sense of classroom experiences and effecting better learning," in Cognition and Instruction: Twenty-Five Years of Progress, edited by S. M. Carver and D. Klahr (Lawrence Erlbaum and Associates, Mahwah, NJ, 2001) Chap, 4.

${ }^{16}$ J. Minstrell, "Teaching science for understanding," in Toward the Thinking Curriculum: Current Cognitive Research, 1989 ASCD Yearbook, edited by L. B. Resnick and L. E. Klopfer (Association for Supervision and Curriculum Development, Washington, DC, 1989) Chap. 7. ${ }^{17}$ J. Minstrell, "Explaining the 'at rest' condition of an object," Phys. Teach. 20, 10-14 (1982). ${ }^{18} \mathrm{~J}$. Minstrell, "Conceptual development research in the natural setting of a secondary school classroom," in Education in the 80's, edited by M. B. Rowe (National Education Association, Washington, DC, 1982), Chap. 9.

${ }^{19}$ P. W. Hewson and M. G. Hewson, "The status of students' conceptions," in Research in Physics Learning: Theoretical Issues and Empirical Studies, edited by R. Duit, F. Goldberg, and H. Niedderer (Institute for Science Education, University of Kiel, Kiel, Germany, 1992), pp. 59 73.

${ }^{20}$ D. I. Dykstra, Jr., "Why teach kinematics?: an examination of the teaching of kinematics and force-I," $\leq$ www.boisestate.edu/physics/dykstra/WTK1.pdf>.

${ }^{21}$ R. R. Hake, "Interactive-engagement vs traditional methods: A six-thousand-student survey of mechanics test data for introductory physics,” Am. J. Phys. 66 (1), 64 - 74 (1999).

${ }^{22}$ D. Hestenes, M. Wells, and G. Swackhamer, "Force concept inventory," Phys. Teach. 30 (3), 141-166 (1992).

${ }^{23}$ D. R. Sweet, "Using microcomputer-based laboratory and constructivist educational theory to teach kinematics to sixth graders," unpublished Masters project, Boise State University (1991). ${ }^{24} \mathrm{We}$ are indebted to a colleague, Robert Bauman for this designation. 
This is an author-produced, peer-reviewed version of this publication. The final, definitive version of this document can be found online at the American Journal of Physics (doi: 10.1119/1.3090824) published by American Institute of Physics, at: http://www.kzoo.edu/ajp/4. Copyright restrictions may apply.

${ }^{25}$ Dykstra and his colleague, Wm. Smith at Boise State University, have tried many different wordings of these instructions to get college students to make the desired motions from the written directions. We have not found a wording that gives satisfactory results. One interactive strategy dealing with the challenge has been found involving interacting with students over these specific instructions.

${ }^{26}$ Dykstra has since developed materials allowing the college students to engage in both methods of resolution, via reasoning from data collected involving different constant forces and reasoning from an experiment in which the force on the cart decreases. The result is a greater number of students changing their notions toward a more Newtonian-like view of force and its relation to motion. $^{20,27}$

${ }^{27}$ D. I. Dykstra, Jr., "Why teach kinematics?: An examination of the teaching of kinematics and force II," <www.boisestate.edu/physics/dykstra/WTK2.pdf $>$.

${ }^{28}$ Because the focus was on linear motion or at least motion along a path, the issue of changing direction was not in contention. The vector nature of physical quantities is yet another conceptual issue. $^{29}$

${ }^{29}$ J. Aguirre and G. L. Erickson, "Students' conceptions about the vector characteristics of three physics concepts,” J. Res. Sci. Teaching 21 (5), 439-457 (1984).

${ }^{30}$ There is some evidence that the existence of this snapshot view of motion may contribute to other problems later in learning about physics. A colleague, Jerry Touger, has reported evidence of using this view in misinterpreting physics they are "taught" in problems they encounter involving motion through changing conditions.

${ }^{31}$ D. E. Trowbridge and L. C. McDermott, "Investigation of student understanding of the concept of acceleration in one dimension,” Am. J. Phys. 49 (3), 242-253 (1981).

${ }^{32}$ We find pressing students still harder to follow directions usually has a negative effect on their thinking as it suggests there is something else they are already supposed to know. In response many students tend to quit trying to make sense and start trying just to guess at things. Not only is this response an too frequent outcome, but many students learn powerfully inappropriate lessons about themselves and about physics, while not changing their own understanding of the phenomena.

${ }^{33}$ Powerful Ideas in Physical Science-A Model Course (American Association of Physics Teachers, College Park, MD, 2003). 
This is an author-produced, peer-reviewed version of this publication. The final, definitive version of this document can be found online at the American Journal of Physics (doi: 10.1119/1.3090824) published by American Institute of Physics, at: http://www.kzoo.edu/ajp/4. Copyright restrictions may apply.

34 T. Wood, P. Cobb, and E. Yackel, "Change in teaching mathematics: A case study,” Am. Educ. Res. J. 28 (3), 587-616 (1991).

${ }^{35}$ References 34 and 36-38 give good examples of the intelligent handling of issues in mathematics and science by children.

${ }^{36}$ D. Schifter and C. Fosnot, Reconstructing Mathematics Education: Stories of Teachers Meeting the Challenge of Reform (Teachers College Press, New York, 1993).

${ }^{37}$ K. Gallas, Talking Their Way Into Science: Hearing Children's Questions and Theories, Responding with Curricula (Teachers College Press, New York, 1995).

${ }^{38}$ P. Cobb, "Reconstructing elementary school mathematics," Focus on learning problems in mathematics 13 (2), 3-22 (1991).

${ }^{39}$ The Flesch-Kincaid Grade Level (FKGL) formula gives the U.S. school reading level by grade of a passage. It can be computed using the formula: $\mathrm{FKGL}=(0.39 \mathrm{X}$ ASL $)+(11.8 \times \mathrm{ASW})-$ 15.59, where ASL is the number of words divided by the number of sentences and ASW is the number of syllables divided by the number of words. FKGL is the readability score calculated in word processing software. 


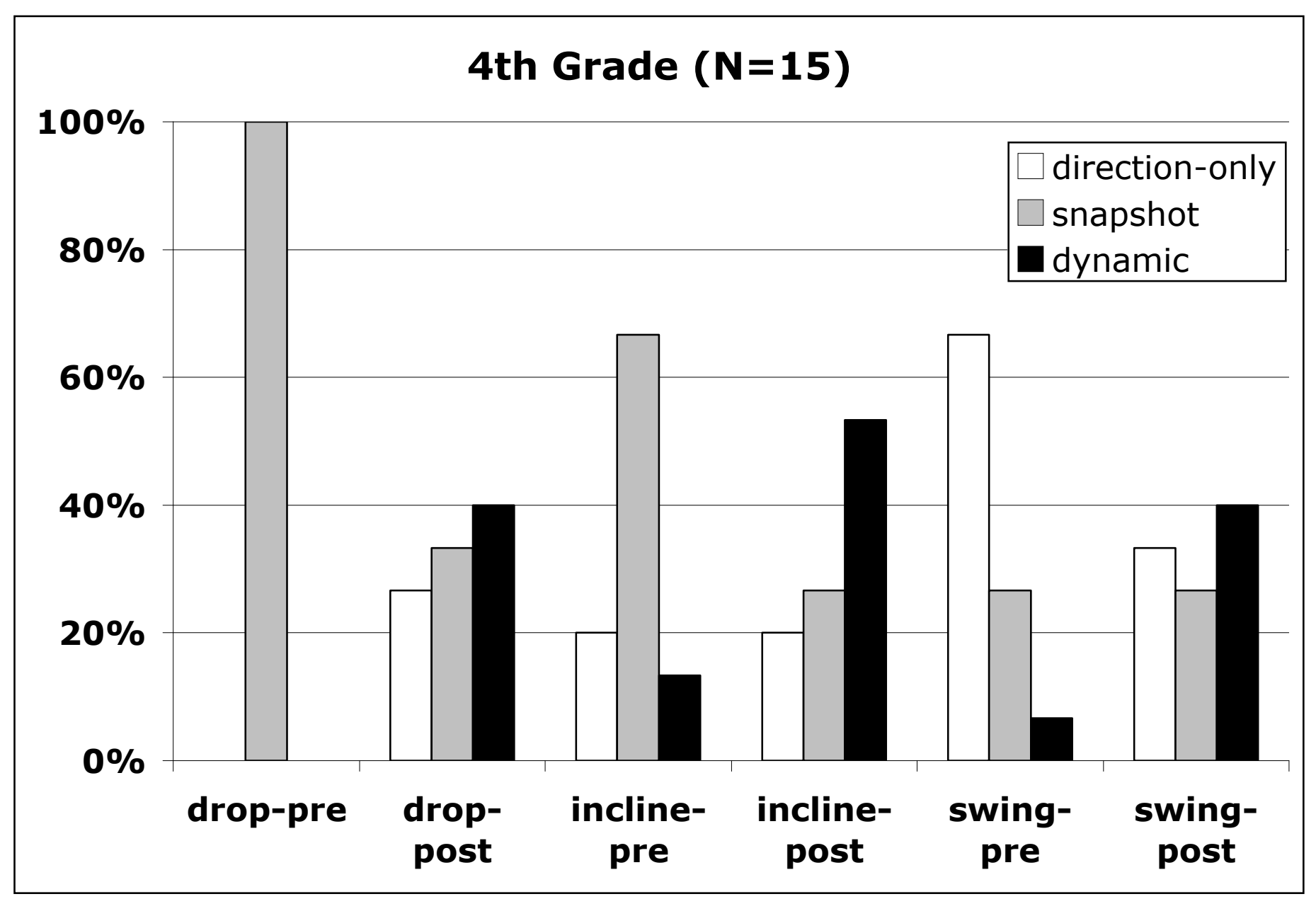

Figure 1 


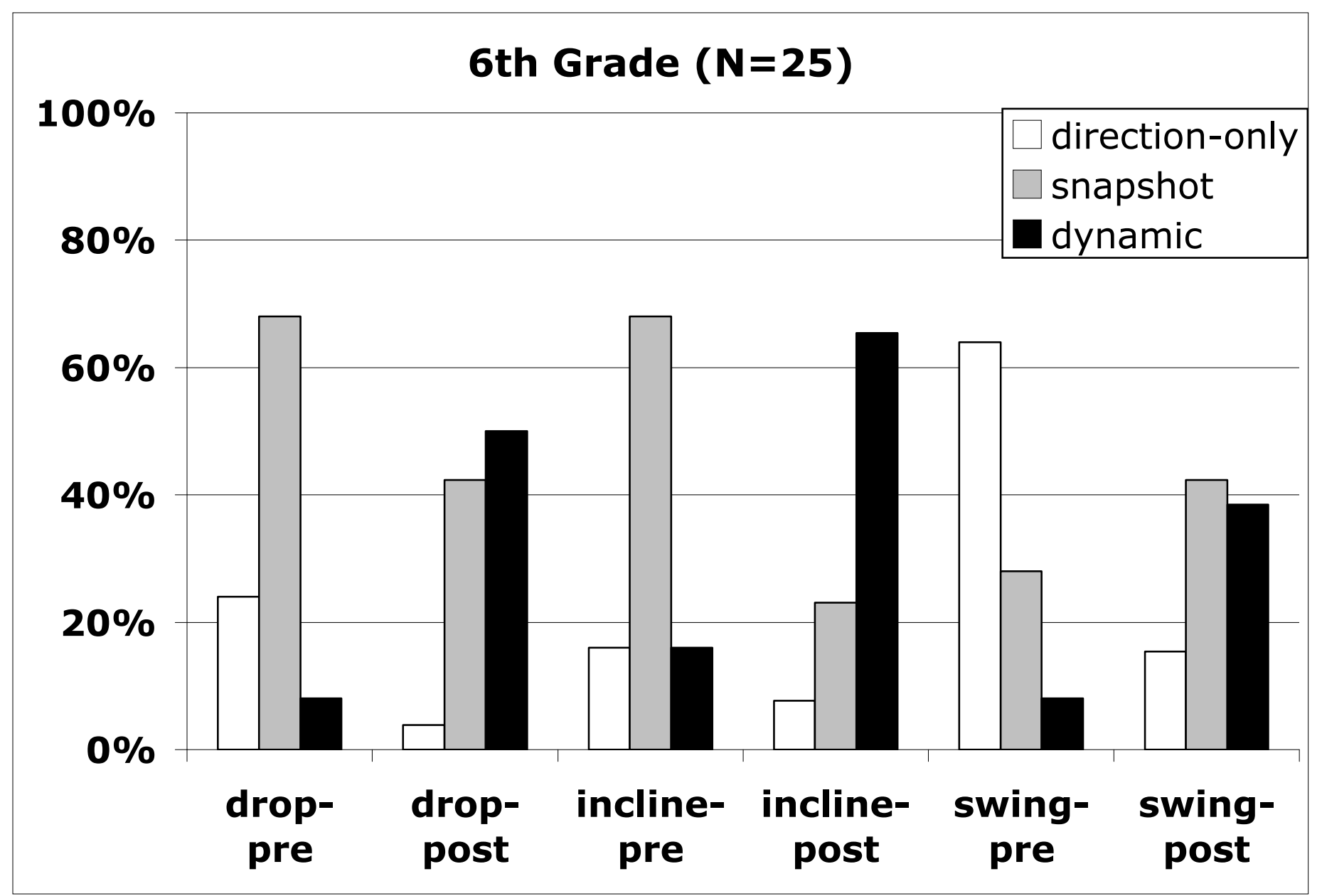

Figure 2 


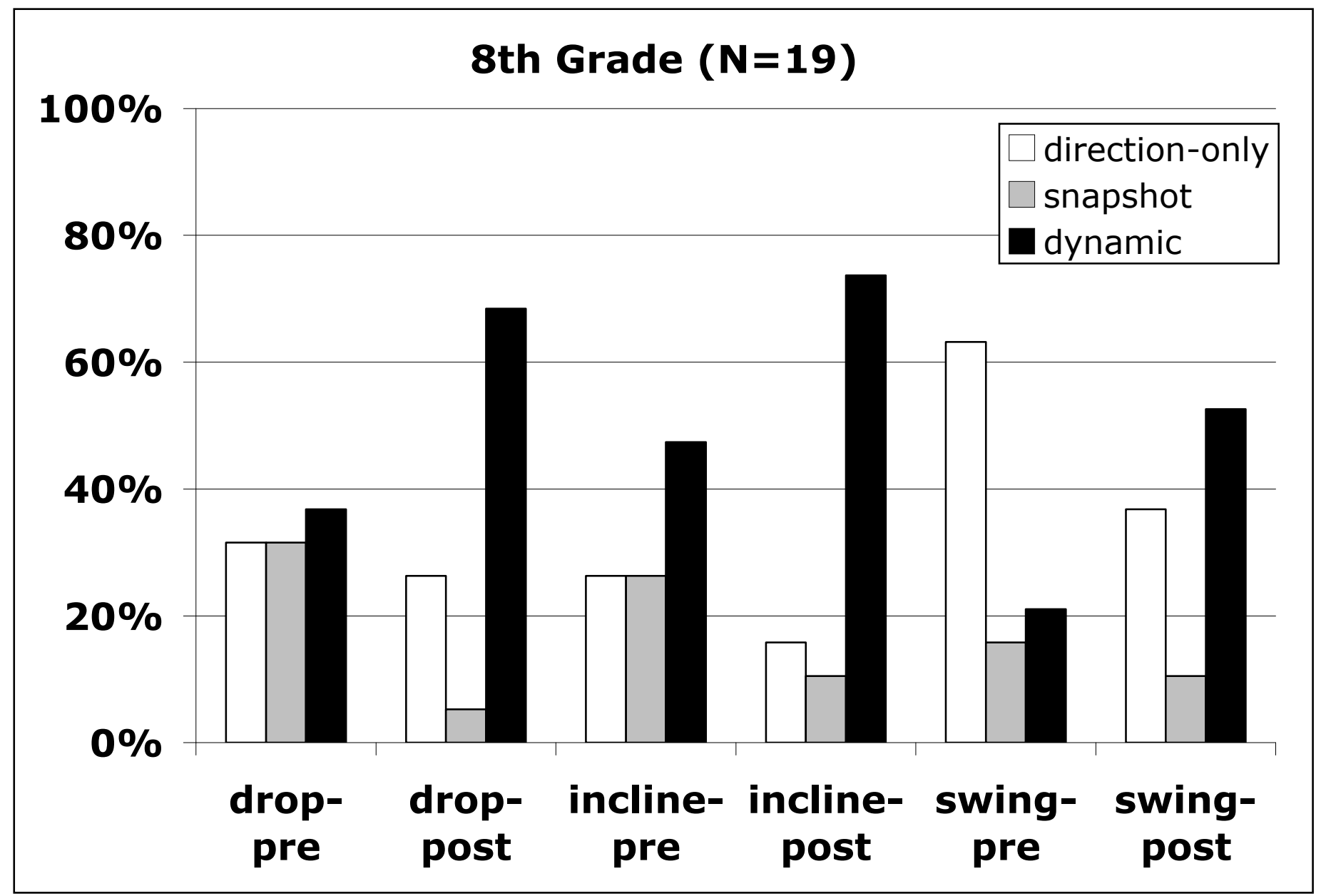

Figure 3 


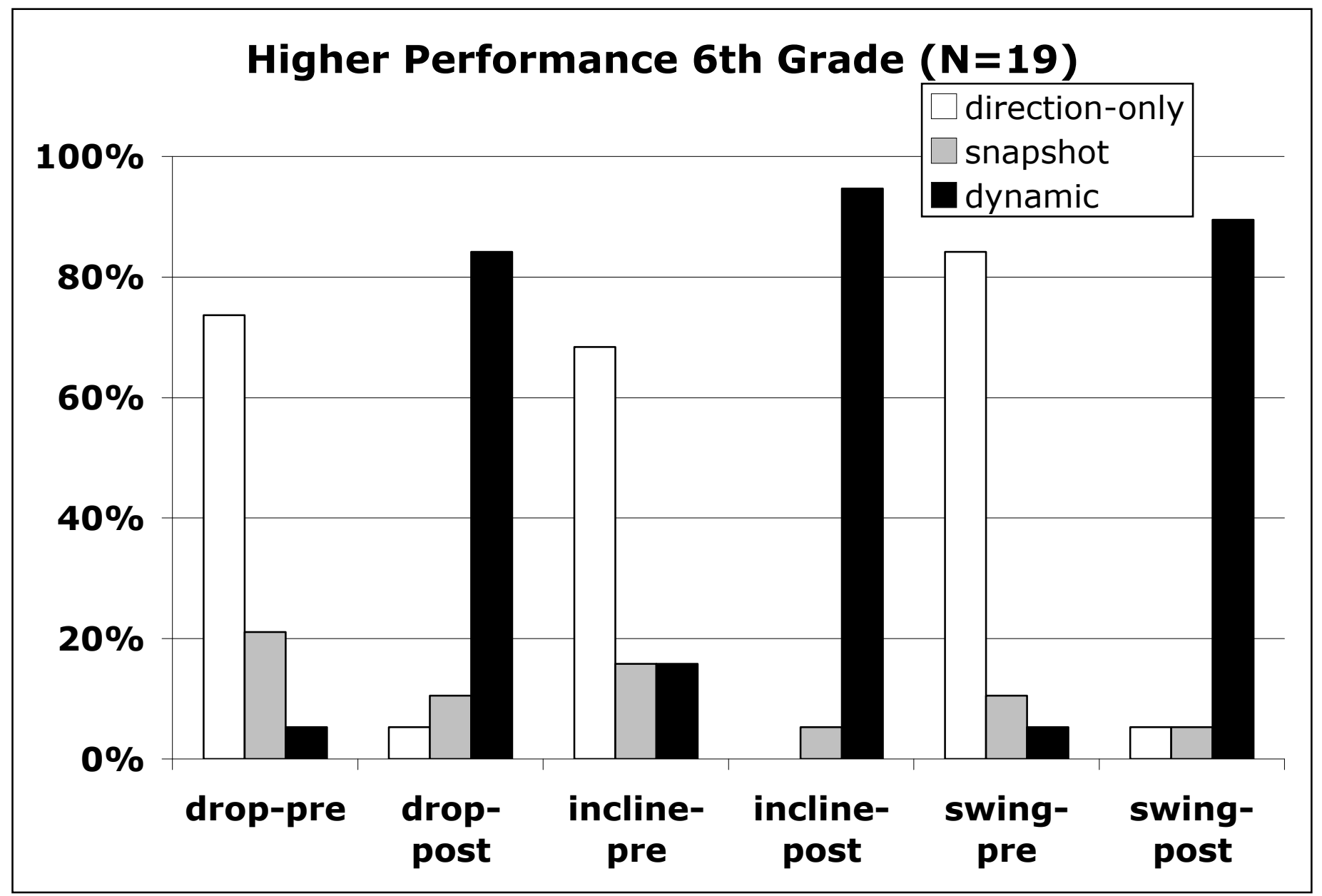




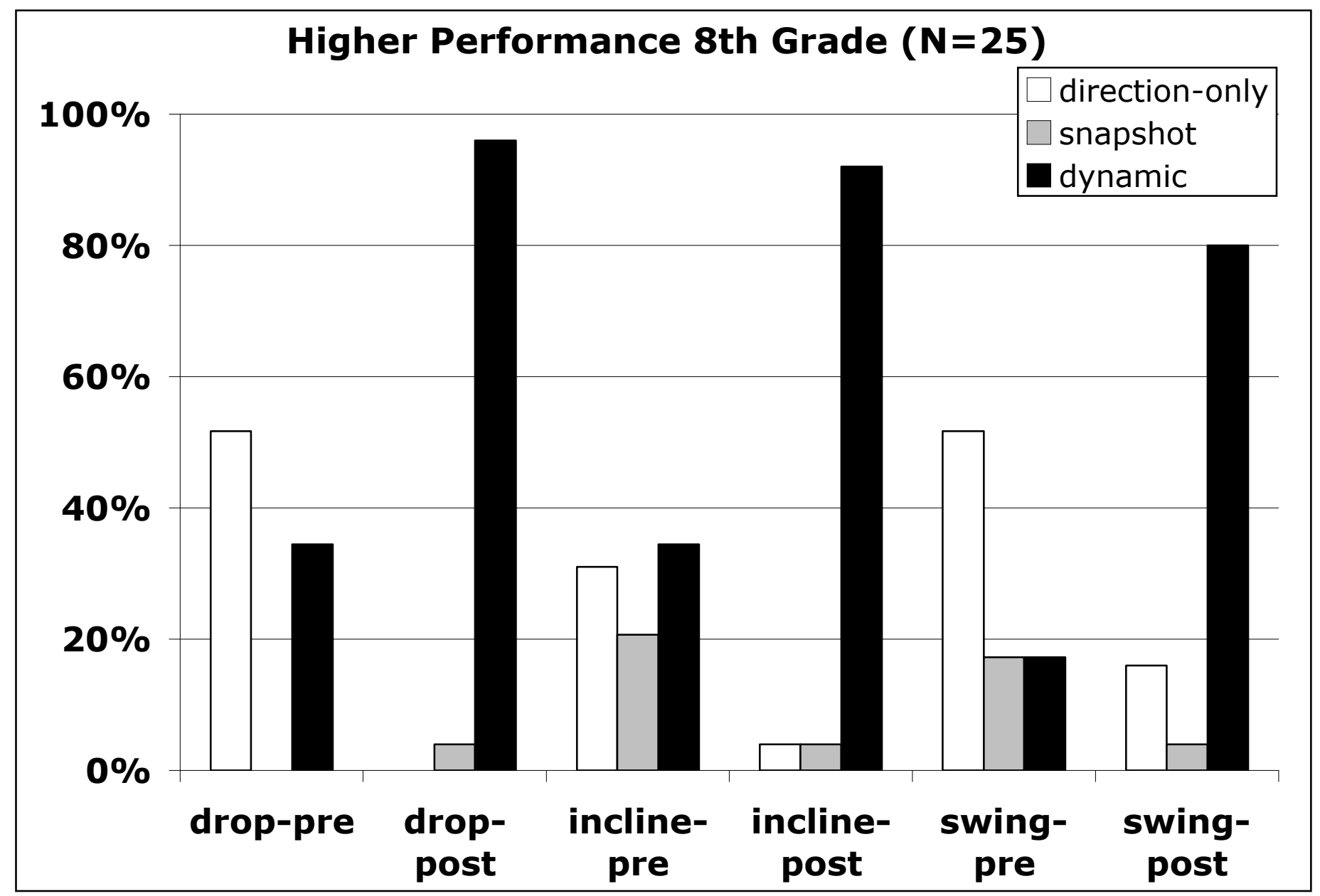

Figure 5 
This is an author-produced, peer-reviewed version of this publication. The final, definitive version of this document can be found online at the American Journal of Physics (doi: 10.1119/1.3090824) published by American Institute of Physics, at: http://www.kzoo.edu/ajp/4. Copyright restrictions may apply.

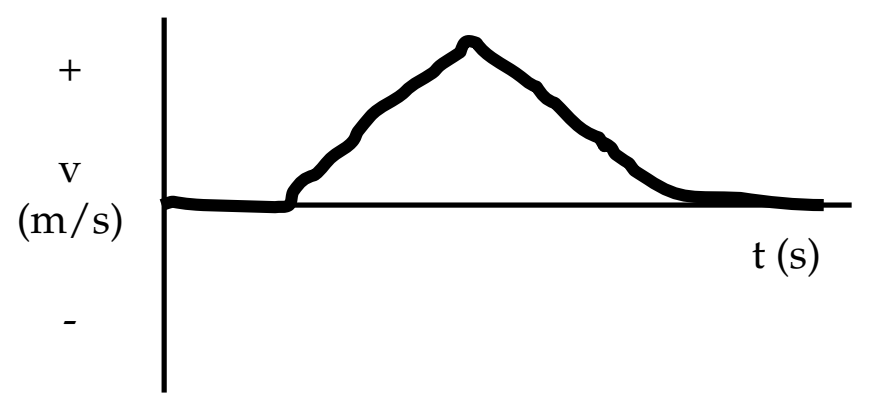

Figure 6(a) 
This is an author-produced, peer-reviewed version of this publication. The final, definitive version of this document can be found online at the American Journal of Physics (doi: 10.1119/1.3090824) published by American Institute of Physics, at: http://www.kzoo.edu/ajp/4. Copyright restrictions may apply.

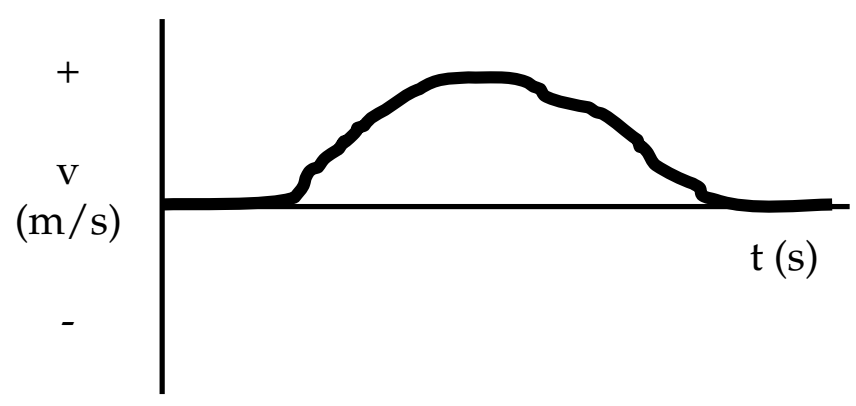

Figure 6(b) 
This is an author-produced, peer-reviewed version of this publication. The final, definitive version of this document can be found online at the American Journal of Physics (doi: 10.1119/1.3090824) published by American Institute of Physics, at: http://www.kzoo.edu/ajp/4. Copyright restrictions may apply.

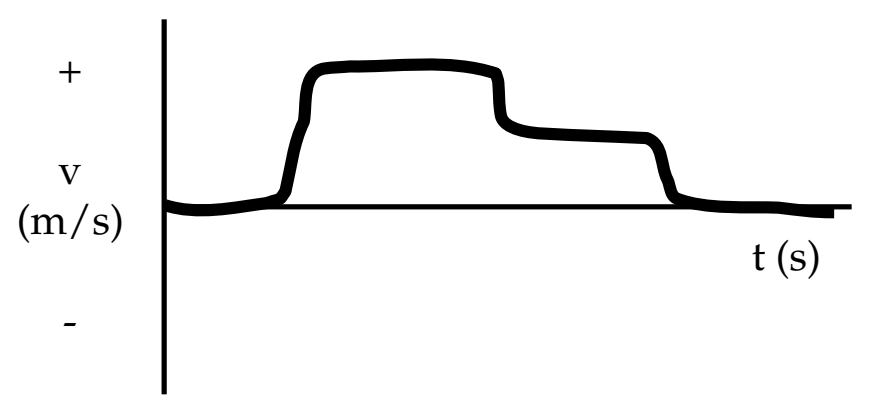

Figure 6(c) 
This is an author-produced, peer-reviewed version of this publication. The final, definitive version of this document can be found online at the American Journal of Physics (doi: 10.1119/1.3090824) published by American Institute of Physics, at: http://www.kzoo.edu/ajp/4. Copyright restrictions may apply.

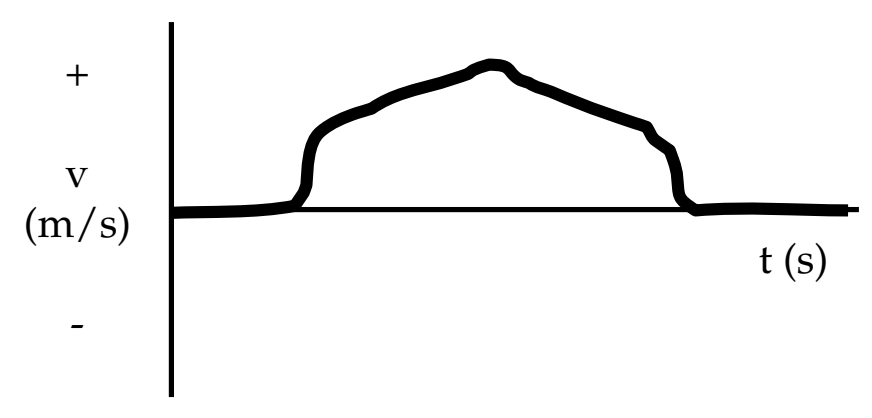

Figure 6(d) 
This is an author-produced, peer-reviewed version of this publication. The final, definitive version of this document can be found online at the American Journal of Physics (doi: 10.1119/1.3090824) published by American Institute of Physics, at: http://www.kzoo.edu/ajp/4. Copyright restrictions may apply.

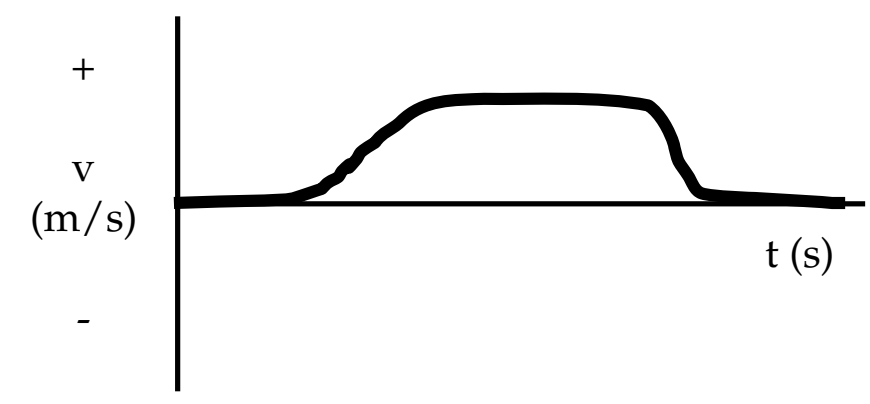

Figure 6(e) 
This is an author-produced, peer-reviewed version of this publication. The final, definitive version of this document can be found online at the American Journal of Physics (doi: 10.1119/1.3090824) published by American Institute of Physics, at: http://www.kzoo.edu/ajp/4. Copyright restrictions may apply.

\section{Figure Captions}

Figure 1: Pre and post diagnostic results for a class of $\mathrm{N}=15$ 4th grade students. The white, gray, and black columns represent the percentage of students giving direction-only, snapshot, and dynamic descriptions of the motion, respectively. For each motion example there is a shift from the direction-only toward the dynamic description of the motion.

Figure 2: Pre and post diagnostic results for a class of $\mathrm{N}=25$ 6th grade students. The columns have the same meaning as in Fig. 1. For each motion example there is a shift from the directiononly toward the dynamic description of the motion.

Figure 3: Pre and post diagnostic results for a class of $\mathrm{N}=19$ 8th grade students. The columns have the same meaning as in Fig. 1. For each motion example there is a shift from the directiononly toward the dynamic description of the motion.

Figure 4: Pre and post diagnostic results for a class of $\mathrm{N}=19$ 6th grade students showing more pronounced change. The columns have the same meaning as in Fig. 1. For each motion example there is an even more pronounced shift from the direction-only toward the dynamic description of the motion than in Fig. 2.

Figure 5: Pre and post diagnostic results for a class of $\mathrm{N}=25$ 8th grade students showing more pronounced change. The columns have the same meaning as in Fig. 1. For each motion example there is an even more pronounced shift from the direction-only toward the dynamic description of the motion than in Fig. 3 . 
Figure 6(a): Predicted velocity-time graph sketched by 15 of 99 non-science college students for the motion described in Sec. VI.B.

Figure 6(b): Predicted velocity-time graph sketched by 21 of 99 non-science college students for the motion described in Sec. VI.B.

Figure 6(c): Predicted velocity-time graph sketched by 40 of 99 non-science college students for the motion described in Sec. VI.B.

Figure 6(d): Predicted velocity-time graph sketched by 9 of 99 non-science college students for the motion described in Sec. VI.B.

Figure 6(e): Predicted velocity-time graph sketched by 6 of 99 college students for the motion described in Sec. VI.B. 
This is an author-produced, peer-reviewed version of this publication. The final, definitive version of this document can be found online at the American Journal of Physics (doi: 10.1119/1.3090824) published by American Institute of Physics, at: http://www.kzoo.edu/ajp/4. Copyright restrictions may apply.

Table 1. Numbers of students in the study. Only those students present for both the pre and the post diagnostic are included in any of the results presented in this report.

\begin{tabular}{c|c|c} 
Semester & Grade & Number of students \\
\hline Spring & 6th & 19 \\
Spring & 8th & 25 \\
Fall & 4th & 15 \\
Fall & 6th & 25 \\
Fall & 8th & 19
\end{tabular}


This is an author-produced, peer-reviewed version of this publication. The final, definitive version of this document can be found online at the American Journal of Physics (doi: 10.1119/1.3090824) published by American Institute of Physics, at: http://www.kzoo.edu/ajp/4. Copyright restrictions may apply.

Table 2: Percentage of students shifting from the snapshot view on the post-diagnostic. $\mathrm{N}$ is the number of students whose descriptions were categorized as snapshot on the pre-diagnostic for each type of motion.

\begin{tabular}{l|c|c|c|c} 
Motion & $\mathrm{N}$ & Snapshot to Dynamic & No change & Snapshot to Direction-only \\
\hline Drop & 41 & $50 \%$ & $33 \%$ & $17 \%$ \\
Incline & 41 & $63 \%$ & $27 \%$ & $10 \%$ \\
Swing & 21 & $48 \%$ & $38 \%$ & $14 \%$
\end{tabular}

